\title{
Humanidades Médicas: ¿Decorativas o Substantivas? El Caso de Literatura y Medicina Humanidades Médicas: Decorativas ou Substantivas? o Caso de Literatura e Medicina
}

\author{
Medical Humanities: Decorative or \\ Substantive? The Case of Literature and \\ Medicine
}

Miguel Kottow

\section{PALABRAS CLAVE}

- Currículo;

- Educación Médica;

- Humanidades Médicas.

PALAVRAS-CHAVE

- Currículo;

- Educação Médica;

- Humanidades Médicas.

\section{KEYWORDS}

- Curriculum;

- Medical Education;

- Medical Humanities.

Recebido em: 21/05/2014

Aprovado em: 31/07/2014

\section{RESUMEN}

Las humanidades médicas han sido proclamadas como esenciales en la educación y en la práctica de la medicina, a pesar de lo cual tienen una posición muy frágil en el currículo médico, siendo consideradas como materias "blandas" frente al currículo nuclear y las asignaturas "duras". El auge de la biomedicina ha enfatizado el carácter cientifista y basado en evidencia, marginando aún más las disciplinas de las humanidades. Basado en la distinción de Whitehead y Latour, entre asuntos fácticos-matters of fact-y asuntos de importancia-importance-o de preocupación-matters of concern. La sociología médica, antropología médica y bioética fundamental (basada en conocimientos de conceptos éticos) logran desarrollar una asignatura con fundamentos empíricos que les permite ingresar al currículo como disciplinas fácticas. Se sugiere una tercera categoría de asuntos de preocupación fáctica-matters of factual concern-para incorporar disciplinas que reflexionan sobre hechos -filosofía e historia de la medicina-, y fomentan la reflexión sobre la medicina, como es la literatura en sus diversas expresiones (narrativa, ensayística, crítica, testimonial). Se propone aquí transmitir esta categoría como conocimiento tácito basado en interacción personal.

\section{RESUMO}

As humanidades médicas foram apregoadas como essenciais na educação e na prática da medicina, embora tenham tido uma posição muito fraca no curriculum médico, sendo consideradas matérias "brandas" em comparação com o currículo nuclear e as disciplinas "duras". O auge da biomedicina enfatizou o caráter cientista baseado na evidência, marginando, ainda mais, as disciplinas das humanidades. Baseado na distinção de Whitehead e Latour, entre assuntos fáticos - matters of fact - e assuntos de importância - importance - ou de preocupação - matters of concern -, a sociologia médica, a antropologia médica e a bioética fundamental (baseada em conhecimentos de conceitos éticos) conseguem desenvolver uma disciplina com fundamentos empíricos que permite a sua entrada no currículo como disciplinas fáticas. O presente trabalho sugere uma terceira categoria de assuntos de preocupação fática - matters of factual concern - para incorporar disciplinas que reflitam sobre fatos - filosofia e historia da medicina -, e fomentem a reflexão sobre a medicina, como é a literatura nas suas diversas expressões (narrativa, ensaística, crítica, testemunhal). A proposta é transmitir essa categoria como conhecimento tácito baseado na interação pessoal.

\footnotetext{
ABSTRACT

Medical humanities have been proclaimed as essential in medical training and practice, yet have achieved no more than a fragile position in medical curricula, being understood as "soft" disciplines in comparison to the core curriculum emphasized by evidence-based biomedicine. Based on Whitehead/Latour's distinction between matters of fact and modes of importance or matters of concern, core curriculum has incorporated medical sociology and medical anthropology as disciplines have been consolidated by empirical knowledge. This paper suggests a third category named "matters of factual concern", which incorporates disciplines that deliberate over facts - philosophy and the history of medicine. Literature also belongs to this category, in its diverse forms of expression: narrative, essayistic, critical and testimonial. Such disciplines are best imparted as tacit knowledge based on personal interaction.
} 


\section{ANTECEDENTES}

\section{DISTINCIONES DICOTÓMICAS}

La explosiva instalación decimonónica de las ciencias naturales desencadenó un pensamiento centrado en desarrollar las diferencias sustantivas entre observación y experimentación del mundo real, dando impulso a un positivismo lógico y empírico, en contraposición con las disciplinas que privilegian la teoría, el método deductivo y el lenguaje cualitativo. Hacia fines del siglo XIX, W. Dilthey presentó su clasificación de ciencias naturales (Naturwissenschaften) y ciencias del espíritu (Geisteswissenschaften). Max Weber diferencia la razón teleológica (Zweckrationalität) de la razón valorativa (Wertrationalität), conceptos que son traídos al pensamiento actual por J. Habermas al referirse a razón instrumental y razón comunicativa, acentuando no solo la divergencia sino la rivalidad entre lo pragmático, destinado a mejorar la adaptación de la vida humana al medio, y los desmedrados esfuerzos de emancipación de la razón comunicativa. C.P. Snow reconoce y lamenta la falta de interlocución entre las dos culturas: ciencia y literatura. El sociólogo Leppenies presenta las ciencias sociales como una tercera cultura. Estas divergencias han invadido el lenguaje cotidiano, como la distinción entre episteme (saber) y doxa (opinión), ciencias duras y disciplinas blandas, investigación cuantitativa o cualitativa, realismo versus constructivismo.

\section{Distinciones Unificadoras}

Tardíamente comienza la reflexión sobre lo reductivo de una clasificación excluyente e incompleta. Ya antes del despliegue de las éticas aplicadas, la bioética en forma predominante, aparecía la sospecha que el lenguaje apropiado para las éticas prácticas no podía sino amalgamar hechos y valores, y dispensar de la falacia naturalista que insiste que una norma no puede derivar de una descripción ${ }^{1}$.

A. N. Whitehead reconoce dos modos de pensamiento, el fáctico -matters of fact-y el modo de importancia -mode of importance- que reflexiona, valora y concede relevancia a la indagación científica de hechos. Destaca que lo fáctico solo gana presencia si la importancia se la concede, un pensamiento que fue adoptado por la sociología de la traducción, muy especialmente por B. Latour, para insistir que los polos divergentes naturaleza/sociedad eran erróneamente enfrentados por cuanto no hay estudio de la naturaleza sin un contexto social $^{2}$. Esta amalgama ha sido reconocida y desarrollada por los Estudios Sociales de Ciencia y Tecnología (ESCT), en el espacio anglohablante denominada Sociology of Science and Technology, (SST).

\section{CIENCIA Y REFLEXIÓN EN MEDICINA}

Desde la segunda mitad del siglo $X X$, se viene desarrollando un fuerte cientifismo en la medicina, enfatizando la práctica médica basada en evidencia, amalgamándola con la ciencia biológica para dar paso a la biomedicina, y estimulando el giro de la investigación clínica al uso de una metódica rigurosa y a la "regla de oro" de los estudios randomizados controlados -Randomized Controlled Trials (RCT) - apoyada por una sólida validación estadística. Llevado a la educación médica, los currículos se diseñan con transmisión de conocimientos técnico-científicos comprobados, el entrenamiento en las competencias a adquirir y aplicar en la práctica médica. Quedan marginadas a segundo orden las disciplinas sociales interesadas en medicina-sociología médica, antropología médica-, y frágilmente incorporadas las humanidades médicas representadas por filosofía, historia, literatura y las artes. La bioética ha logrado entrar en los currículos de las profesiones de la salud, pero ocupa un lugar muy periférico y con tendencia a una progresiva atrición.

Hay un movimiento por recuperar las humanidades para la educación superior ${ }^{3,4}$, y ha tiempo que aparecen regularmente publicaciones periódicas, libros e innumerables artículos interesados en las "humanidades médicas". Todos estos esfuerzos se diluyen y lamentan el escaso impacto que logran en los currículos médicos, debiendo conformarse con el reconocimiento que "las humanidades médicas aún no encuentran su identidad y forma determinadas" ${ }^{\prime 5}$ (p. 33). Señal adicional de fragilidad es la postura que estas disciplinas por ningún motivo deben ser ingresadas al currículo obligatorio, recomendándose que sean cursos "breves, voluntarios, disponibles para quienes tienen este tipo de interés" ${ }^{\prime \prime}$ (p. 37), enfatizando la filosofía y la filosofía de la medicina como esqueleto de las humanidades médicas ${ }^{7}$. Estos minimalismos no son coherentes con la importancia que otros conceden a las humanidades en la formación integral del médico al asignarles la tarea de recuperar la humanización en salud ${ }^{8}$. Algunos intentos enfatizan la necesidad que las humanidades médicas, dada su importancia en desarrollar el "humanismo en medicina", sean componentes obligatorios del currículo médico, y exploran diversos modos didácticos de desarrollar estos temas ${ }^{9}$.

\section{PROPUESTA PARA LAS HUMANIDADES MÉDICAS}

Algunos puntos que animan la presente reflexión:

- Deshacer la división entre disciplinas médicas duras -biomedicina- y las disciplinas así llamadas blandas.

- Introducir la categoría de las materias de importancia fáctica.

- Reconocer la categoría de disciplinas sociales como ya incorporadas al currículo fáctico. 
- Distinguir en las humanidades médicas aquellas que deben integrarse al currículo nuclear, de aquellas que son enriquecedoras pero pueden permanecer como ofertas facultativas.

- Establecer características cognitivas que han de orientar la formación y la reflexión de las humanidades médicas.

La distinción usual entre currículo nuclear y el valor agregado de ciertas disciplinas llamadas blandas ha sido poco propicia al fortalecimiento curricular de las humanidades médicas y debe ser repensada, ventajosamente, utilizando los conceptos de Whitehead/Latour:

\section{Matters of fact}

Biomedicina

Medicina basada en evidencia

Códigos de conducta profesional

\section{Matters of concern} Bioética fundacional Bioética clínica Sociología médica Antropología médica Filosofía de la medicina Historia de la medicina Literatura y medicina

Artes pictóricas, dramáticas, musicales

\section{ASUNTOS FÁCTICOS}

La bioética fundacional es una asignatura que imparte y explica fundamentos de la ética, como los conceptos de utilitarismo, derechos y deberes, principios, valores y virtudes, aseveraciones éticas de intención universalista versus las relativas, contextuales y situacionales. Las Declaraciones de los DD.HH. existen de facto, como también la Declaración de Helsinki, los textos de filosofía y de ética. Conceptos e instrumentos todos que pueden ser motivo de enseñanza objetiva y constituirse en asignatura nuclear. La bioética clínica, en cambio, es materia de reflexión y deliberación que no se imparte sino que se comparte en discusión de casos, resolución de problemas bioéticos, diálogo e interacción.

La sociología y la antropología médicas han desarrollado discursos coherentes, inclusivos de componentes empíricos que permiten una enseñanza expositiva pero que mantienen el carácter de modos importantes de análisis y valoración por cuanto sus fundamentos teóricos son diversos y constructivos. Una visión socio-antropológica es siempre un constructo que no refleja la realidad en sí, sino que el desenvolvimiento de la disciplina y sus cultores, pero tiene un corpus extenso de investigación empírica, de modo que se convierten en disciplinas indispensables para los estudios médicos.

Ha sido más difícil justificar la participación de la filosofía e historia de la medicina, y el cultivo de la literatura en medicina, siendo necesario insistir que la formación médica no puede prescindir de una reflexión sobre enfermedad, enfermabilidad (Laín Entralgo), salud, objetivos y fines de la medicina, reconociendo que el médico no es un reparador mecánico de organismos dañados. Su tarea, realizada indiscutiblemente con rigor técnico-científico, es una interlocución de un potencial terapeuta con una existencia enferma, una población vulnerada, sufriente y susceptible a daños corpóreos, el cuerpo entendido como un organismo para soslayar la distinción problemática entre cuerpo y mente. El médico ha de ser fiel a lo que se solía denominar una "profesión docta", ha de saber que Hipócrates no solo es una figura histórica, sino presente: facie hipocrática, maniobra hipocrática, signos hipocráticos, el autor de la no maleficencia (primum non nocere): que Galeno, además de haber sido médico en el Imperio Romano, se perpetúa con la receta galénica -que vuelve a renacer en la farmacogenética-, y ha dejado su impronta en la denominación del médico como galeno. La historia de los trabajos de Jenner explica, al menos, por qué hoy se habla de vacunas y no de perrunas o gatunas, la relación entre animales y seres humanos reapareciendo en los debates sobre xenotrasplantes. Recordar el descubrimiento de Fleming como acto de serendipia también trae al presente la historia social compleja de la indagación farmacológica. El escándalo de Tuskegee Valley es también la historia de la penicilina y su lento ingreso a la terapéutica médica.
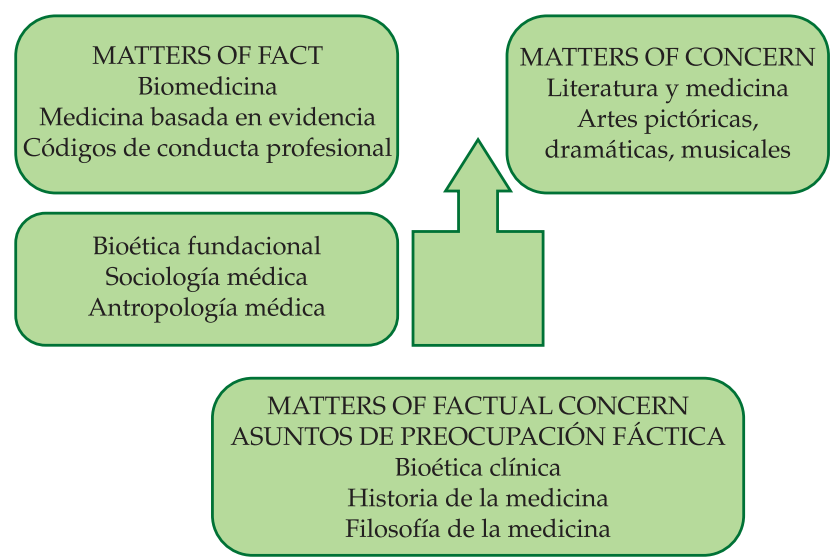

Van en camino de ser fácticas, pero envueltas en presupuestos teóricos, haciendo plausible la introducción de una categoría intermedia entre lo fáctico y lo importante: matters of 
factual concern o asuntos de preocupación fáctica, es decir, modos de pensar e indagar que reconocen una fundamentación empírica que es sometida a reflexión y valoración.

La medicina es una tradicional profesión liberal, denominación que se refiere a su independencia, pero también a la expectativa social de que provea "servicios conceptuales e intelectuales en el interés del cliente y el público ${ }^{10}$. Más allá de sus competencias profesionales, es un referente social para asuntos de salud y ética, y para desempeñarla los médicos requieren un acervo cultural más amplio que el de competencias y habilidades profesionales. El médico en formación ha de tener la capacidad de entender y cultivar la diferencia entre un pensamiento reflexionado en su quehacer y sobre la medicina que ejerce. En forma similar, más que acopiar información histórica, ha de ser capaz de una mirada historiográfica que explique datos y hechos como inmersos en el contexto social en que ocurrieron. De no hacerlo, le será difícil entender la medicina de Albert Schweitzer, las dificultades de Semmelweis para dar a conocer sus hallazgos, el tardío reconocimiento de Ludwik Fleck, la figura de Samuel Hahnemann buscando el modo de curar "juicioso y racionalmente" en la época en que la medicina alopática era pobre, pero que vuelve a ser atractiva ahora que la alopatía sufre la hipertrofia de los excesos. La historia indica cómo "el conocimiento médico no es 'objetivo'; más bien es una función de tiempos y lugares históricos. Imparte algún escepticismo sobre prácticas médicas actuales"11 (p. 77).

Filosofía en medicina e historia de la medicina son materias que deben preocupar al médico para el mejor desempeño de su rol profesional y social, siendo asuntos que tienen aspectos fácticos así como pertinencia y relevancia, de donde viene la propuesta de incorporarlas al currículo nuclear como asuntos de preocupación fáctica. Pero no basta saber algo de filosofía y medicina, es necesario lograr la reflexión activa sobre diversos tópicos: la orientación de la medicina contemporánea: medicina del deseo, pacientes-sanos, prevención personal autoresponsable en base a una epidemiología de riesgo poblacional, el énfasis en determinantes genéticos, la marcha hacia una farmacogenética que, de ser exitosa, con toda probabilidad ampliará la brecha y el acceso.

\section{LITERATURA Y MEDICINA}

La literatura relacionada con la medicina quedó, junto con las artes, como una materia de importancia que prescinde de datos y cuantificación, pese a la "presunción a favor de la centralidad de literatura o filosofía"12 (p. 2). No obstante, es conveniente distinguir entre el rol nuclear, aunque no fáctico, que la literatura adopta en la formación médica, a diferencia de las diversas artes que sin duda enriquecen a la persona, pero lo hacen de acuerdo a ciertas inclinaciones individuales. La afinidad por la música puede faltar sin empobrecer al futuro médico quien, tal vez, vea en el teatro una fuente valiosa y grata de cultivar.

- El cultivo de la literatura en los estudios médicos se candidata a incorporarse como materia curricular obligatoria, dada su capacidad irremplazable como vehículo de imágenes, saberes, críticas y testimonios. Este argumento gana en plausible fuerza si se presenta una distinción de los diversos modos literarios que existen:

- La literatura narrativa, que relata diversas formas que ha tenido la práctica médica a lo largo del tiempo, y que han desaparecido del ejercicio biomédico contemporáneo: el médico de cabecera, la visita domiciliaria, como se reflejan en narrativas de Tolstoi, Chejov, Kafka.

- La literatura ensayística, orientada al despliegue de ideas sobre enfermedad y el quehacer de la medicina, como se encuentra en Canguilhem, Césarman, Sontag, Laín Entralgo, Marañón.

- El texto crítico, que denuncia formas defectuosas de la medicina: Illich, Berlinguer, Ongaro Basaglia.

- Los relatos testimoniales que retratan el mundo subjetivo del paciente -Cousins, Frank- y la actividad del médico-Manheimer-.

\section{CONOCIMIENTO EXPLÍCITO Y CONOCIMIENTO TÁCTICO}

Uno de los más serios problemas con que se enfrenta la filosofía de la educación es el método por mantener un balance adecuado entre lo informal y lo formal, lo incidental y lo intencional, en los modos de educación. Cuando la adquisición de información y de habilidades técnicas intelectuales no tiene influencia sobre la formación de una disposición social, la experiencia vital ordinaria deja de ganar en sentido, mientras que la formación escolar, hasta allí, solo crea "agudos" -sharps-en aprendizaje, es decir, especialistas egoístas ${ }^{13}$ (p. 11).

Es habitual hablar de la transmisión de conocimientos no explícitos como currículo oculto, concepto que tiene algunas connotaciones negativas: el currículo oculto no es necesariamente curricular cuando se refiere a actividades que socializan y transmiten valores en la vida cotidiana del alumno; la denotación de oculto es precisamente contraria a lo que en educación se busca transmitir "desocultando" -develandovalores, actitudes, tradiciones. El currículo oculto es con fre- 
cuencia visto como un valor negativo que destila ideologías que no sea desea explicitar, pero sí transmitir implícitamente.

Hay numerosos intentos de enseñar las humanidades médicas, desde clases expositivas, grupos de discusión, hasta integración transversal de programas de resolución de problemas, talleres, reuniones informales. La conclusión frecuente es que una estructuración formal del currículo de las humanidades médicas suele tener escasa acogida entre los estudiantes, mientras que la integración transversal diluye y limita el espectro de las humanidades, suele no cumplir los objetivos propuestos y termina por reducirse a pinceladas de ciencias sociales, de salud poblacional, de "medicina psicológica"14.

Michael Polanyi introdujo el concepto de conocimiento tácito en distinción del explícito. El conocimiento explícito es fáctico, expresado, formalizado, sistematizado, a diferencia del conocimiento tácito que "tenemos sin saber que lo tenemos": no es expresable, pues si lo fuese, dejaría de ser tácito, pero es transmisible a través de relaciones interpersonales como el diálogo, la tutoría, el comportamiento ejemplar ${ }^{15}$.

Si se reconoce a las humanidades médicas como asuntos de preocupación fáctica, que cabalgan entre la enseñanza formal y la transmisión tácita, por una parte conteniendo conocimientos explícitos pasible de ser expresados, informados y transmitidos, quedando, por otra parte el componente de conocimientos tácitos que por definición no pueden ser expresados, pero sí entregados, compartidos entre quienes poseen ese conocimiento tácito referido a la medicina y quienes buscan adquirirlo, reemplazando la enseñanza de puramente expositiva, o al menos enriqueciéndola con espacios de reflexión, el método llamado "socrático", la deliberación, la tutoría.

Confiar las humanidades médicas requiere docentes apropiados. El contacto personal que permita transmitir lo que ahora se denominan "competencias blandas" no puede darse entre expertos que se acercan desde afuera a la medicina y que podrán ser competentes expositores de conocimiento explícito e incluso eficaces entrenadores en los aspectos formales de disciplinas como comunicación, informática, el marco general de las disciplinas sociales. Pero el conocimiento tácito del médico que, como decía el decimonónico clínico español J. Letamendi, "si solo sabe medicina, ni medicina sabe", la transmisión de la compleja relación existencial con el enfermo, y la prestancia de opinar confiablemente en materias de medicina a nivel social, solo se podrá transmitir por quienes efectivamente viven la medicina en forma reflexiva, conocedora de su pasado y capaz de forjarse una presencia profesional que enfrente las reducciones y limitaciones que los sistemas, los determinantes socioeconómicos y los poderes fácticos llevan a la medicalización, a la tecnificación y a la economización de la medicina.

\section{CONCLUSIÓN}

La importancia en las humanidades médicas ha sido presentada en múltiples ocasiones y con los más variados argumentos. Las escuelas de medicina tienen la doble tarea de "servir a al público [la sociedad], así como de cultivar la opinión pública"16 (p. 76). Que la autorizada voz de E. Pellegrino ${ }^{17}$, entre muchos otros, haya recalcado que "para curar a otra persona debemos comprender cómo la enfermedad lesiona su humanidad" (p. 256), debe ser motivo de preocupación, porque lo señalado hace 30 años no ha logrado inscribirse en la enseñanza ni en la práctica médica. Cualquier deliberación al respecto no hace más que confirmar lo tantas veces dicho, lo que este texto pretende es discurrir un modo de insertar con firmeza aquello que todos respetan pero pocos logran hacer realidad. El impulso por conquistar espacios curriculares nucleares conlleva el riesgo que allí predomine una atmósfera utilitarista de corto alcance y profesionalismo estrecho ${ }^{18}$.

El resumen de una publicación sobre desarrollo curricular señala 10 puntos esenciales a considerar y los "ilustra mediante la analogía de la construcción de automóviles". Muy hollado, pero del todo relevante, es volver sobre la $11^{a}$. Tesis de Contra Feuerbach: "Los filósofos han reflexionado sobre el mundo, ahora es tiempo de modificarlo".

\section{REFERENCIAS}

1. Putnam H. El desplome de la dicotomía hecho-valor y otros ensayos. Barcelona: Paidós; 2004.

2. Latour B. Nunca fuimos modernos. Buenos Aires: Siglo XXI Eds.; 2007.

3. Nussbaum MC. Cultivating Humanity. Cambridge London: Harvard University Press; 2000.

4. Small H. The value of humanities. Oxford: Oxford University Press; 2013.

5. Pattison S. Medical Humanities: a vision and some cautionary notes. Med Humanities 2003; 29:33-36.

6. Downie RS. Medical humanities: a vision and some cautionary notes. Med Humanities 2003; 29:37-38.

7. Louhiala P. Philosophy for medical students -why, what, and how. Med Humanities 2003; 29:87-88.

8. Lima CC, Martinez Guzman S., Craice De Benedetto MA, Claramonte Gallian DM. Humanidades e humanização em saúde. Interface 2014; 18(48):139-150.

9. Ayres JRCCM, Rios IC, Falcão MTC, Nota A. Humanidades como Disciplina de Graduação em Medicina. Revista Brasileira de Educação Médica 2013; 37(3):455-463.

10. Directive on Recognition of Professional Qualifications: 2005/36/EC.

11. Lerner 2005, citado en Halperin 2010, (nota 16). 
12. Evans HM, Grwaves DA. Looking for emerging themes in medical humanities -some invitations to our readers. Medical Humanities 2003;29:1-3.

13. Dewey J. Democracy and Education? [s.1]: WLC Books; 2009.

14. Chiavaroli N, Ellwood C. The Medical Humanities and the Perils of Curricular Integration. J Med Humanit 2012; 33:245-254.

15. Hildreth PM, Kimble C. The duality of knowledge. Information Research, 2002; 8(1) [Accedido el 13 de abril de 2014]. Disponível em: http://InformationR.net/ir/8-1paper142.html.

16. Halperin EC. Preserving the humanities in medical education. Medical Education 2010;32:76-79
17. Pellegrino ED. The humanities in medical education: Entering the post-evangelical era. Theor Med Bioeth 1984; 5:253-266

18. Harden IRM. Ten questions to ask when planning a course or curriculum. Medical Education 1986; 20(4):356-365

\section{CONFLICTO DE INTERESES}

El autor declara no tener conflicto de intereses alguno.

\section{DIRECCIÓN PARA CORRESPONDENCIA}

Miguel Kottow

José Miguel de la Barra, 521 - Depto. 4-B

Santiago - Chile

E-mail: mkottow@gmail.com; mkottow@med.uchile.cl 